\title{
La aplicación del método AICLE en los estudios de comunicación de la Universidad de Vic
}

\author{
Xavier Ginesta PoRTET \\ Universitat de Vic \\ xavier.ginesta@uvic.cat \\ Gerard COLL-Planas \\ Universitat de Vic \\ gerard.coll@uvic.cat \\ Jordi De SAn Eugenio Vela \\ Universitat de Vic \\ jordi.saneugenio@uvic.cat
}

Recibido: $19 / 11 / 2012$

Aceptado: 23/01/2013

\section{Resumen}

En este artículo se elabora un diagnóstico de las asignaturas que siguen el método AICLE (Apredizaje Integrado de Contenidos y Lenguas Extranjeras) en la Facultad de Empresa y Comunicación (FEC) de la Universitat de Vic (UVic). En base a cuatro entrevistas semiestructuradas al profesorado que las imparte y a encuestas a 60 estudiantes de segundo ciclo de los estudios de comunicación, se elabora un diagnóstico de los puntos fuertes y débiles de la aplicación de este método y se recogen propuestas para mejorar el aprendizaje del inglés en las carreras de Periodismo, Comunicación Audiovisual y, también, de Publicidad y Relaciones Públicas que se imparten en la Universitad de Vic.

Palabras clave: Inglés, evaluación, comunicación, educación, AICLE

\section{The application of CLIL in the media studies of the University of Vic}

\begin{abstract}
The authors of this article elaborate a diagnostic of the CLIL courses in the Faculty of Business and Communication (FEC) of the University of Vic (UVic). Authors have done in-depth interviews with four CLIL lecturers of the faculty, as well as, a survey using 60 students of 3rd and 4th course of media studies. Using this data, these researchers elaborate a diagnostic of the application of CLIL, highlighting pros and cons of the method and recollecting different proposals to improve the application of this methodology in the bachelors of Journalism, Audio-visual Communication and, finally, Advertising and Public Relations.
\end{abstract}

Key words: English, evaluation, media studies, education, CLIL

\section{Referencia normalizada}

GINESTA PORTET, Xavier; COLL-PLANAS, Gerard; y DE SAN EUGENIO, Jordi (2013): "La aplicación del método AICLE en los estudios de comunicación de la Universidad de Vic". Estudios sobre el mensaje periodístico. Vol. 19, núm. especial abril, págs.: 813-821. Madrid, Servicio de Publicaciones de la Universidad Complutense.

\section{Sumario}

1. Introducción; 1.1. Objetivos; 1.2. Estado de la cuestión. El método AICLE en el nuevo Espacio Europeo de Educación Superior. 2. Metodología. 3. Desarrollo. Los estudios de Ciencias de la Comunicación de la UVic y la aplicación del AICLE; 3.1. Beneficios del AICLE; 3.2. Inconvenientes del AICLE; 3.3. Soluciones y aspectos a mejorar. 4. Conclusiones. 5. Referencias bibliográficas. 


\section{Introducción ${ }^{1}$}

Desde la implementación de los grados de comunicación, hace ya cuatro cursos académicos, la UVic ha sido especialmente estricta en la enseñanza de la lengua inglesa -obligando a todo el alumnado a acabar sus estudios con un nivel B2-, tanto a través de asignaturas propiamente de lengua inglesa (15 ECTS obligatorios), como asignaturas de contenido impartidas en inglés siguiendo el método AICLE. Éstas se ofrecen conjuntamente a estudiantes internacionales (ERASMUS o de intercambio bilateral) y nacionales, de la misma universidad o del programa SICUE. El curso 2011-12 ha significado la implementación total de las asignaturas AICLE en los grados de Ciencias de la Comunicación, por lo que es en este momento que se puede plantear un primer análisis y valoración de como han funcionado para poder mejorar sus planteamientos en su segundo año de impartición.

\subsection{Objetivos}

El objetivo general de esta investigación es describir el panorama de la enseñanza impartida en inglés (AICLE) con la que se encuentran los alumnos y profesores de tercer y cuarto curso de los grados de Ciencias de la Comunicación de la FEC, y la evaluación que hacen de esta. De este objetivo general se desprenden varios objetivos específicos:

» Identificar los obstáculos que tienen los alumnos que no optan por cursar asignaturas de contenidos en inglés.

»Elaborar propuestas de mejora en base a las valoraciones de los profesores y los estudiantes, tanto los que han cursado estas asignaturas como los que no.

»Descubrir si la percepción e identificación de problemas es la misma entre los profesores y los alumnos.

» Determinar diferencias en la adquisición de conocimientos entre los estudiantes que han cursado estas asignaturas y los que no.

\subsection{Estado de la cuestión. El método AICLE en el nuevo Espacio Europeo de Educación Superior}

En el contexto catalán, donde se circunscribe esta investigación, desde 1995 se promovió un plan de impulso de lenguas extranjeras en la Educación Primaria (Vallbona, 2008) que consistía en formar a los maestros. Así comenzaron estos proyectos experimentales de integración de idioma y contenido que en 2012 comienzan a integrarse en Bachillerato y en las titulaciones universitarias.

La implantación de este nuevo sistema de aprendizaje no se podrá valorar completamente hasta que los estudiantes que han empezado a convivir con él en la Educación Primaria no lleguen a la universidad. Será entonces cuando se podrá llegar a conclusiones fidedignas y valorar si el nivel de inglés ha mejorado o no en los estudiantes universitarios.

1 Este estudio ha sido elaborado a partir de un trabajo de investigación realizado en la asignatura de Métodos de Investigación en Comunicación por estudiantes de $3 \mathrm{r}$ curso de Periodismo. Un agradecimiento especial para los estudiantes María Fernández, Crístian Franco, Xavier Freixa y Sergi Escudero. 
Los motivos del reciente interés en el AICLE pueden explicarse desde dos perspectivas: un cambio de mentalidad en lo referido a las formas más efectivas de aprendizaje de idiomas y el proceso de integración europea, que ha generado la necesidad de mejorar el nivel de lenguas extranjeras (Marsh y Largé, 1999; Marsh, Marsland y Maljers, 1998; Marsh, 2008; Coyle, Hood y Marsh, 2010).

Actualmente los estudiantes tienen muy interiorizados conceptos formales, sobre todo vinculados a aspectos gramaticales, pero tienen obstáculos a la hora de utilizar el idioma en entornos comunicativos. En este sentido, Marsh (2008) considera que en el aprendizaje de idiomas debe prestarse especial atención a la definición de competencias adquiridas de forma objetiva, superando la "educación formal" para adentrarse de lleno en el uso del idioma (Marsh, 2008).

Existe, por tanto, una concepción en cierto modo obsoleta sobre el aprendizaje de idiomas, que en España es más notoria debido al bajo nivel de conocimiento de lenguas extranjeras: el país ocupa el lugar 24 en el ránquing de conocimiento del inglés, entre los países de lengua no inglesa (EF EPI, 2009).

Concretamente, el AICLE se refiere a cualquier contexto de aprendizaje en el que el contenido y el idioma están integrados para dar respuesta a objetivos educativos específicos. Por un lado, podría utilizarse para referirse a una clase en la que un profesor de lengua extranjera enseña a sus alumnos un contenido no vinculado al idioma, en una lengua extranjera. Por otro lado, también puede aplicarse a una situación en la que el profesor de una asignatura cualquiera utiliza un idioma extranjero como medio de instrucción de una lección concreta (Marsh, 2008). Este es el caso que aquí nos ocupa. Coyle (2005) propone la impartición de clases en método AICLE en base a cuatro 'c':

»Contenido. Se refiere al progreso en el conocimiento, a las técnicas usadas para entender el contenido de un currículum impartido en inglés.

" Comunicación. El lenguaje se utiliza para aprender y, a la vez, para comunicarse.

\Cognition (procesos cognitivos). El alumno desarrolla técnicas y capacidades efectivas en la lengua materna y extranjera, de una forma abstracta y concreta.

»Cultura. Es uno de los elementos más necesarios e importantes del programa bilingüe. Aprender una lengua es también la cultura donde ésta ha nacido, así como otros valores culturales y puntos de vista.

En lo referido a la evaluación del AICLE, resulta fundamental la priorización, por parte de los docentes, de la comunicación y la fluidez, en lugar de la exactitud gramatical. Es por ello que se desarrollan métodos normalizados de evaluación que reconozcan y premien las habilidades de comunicación con preferencia sobre la enseñanza memorística y la destreza gramatical. Según Álvarez (en Álvarez et al., 2009) la enseñanza bilingüe que integra lengua y contenidos dispone de un gran potencial pero no hay que establecer unos principios dogmáticos rígidos: la aplicación del método se debe adaptar al contexto.

Vallbona (2008), en base a su investigación, afirma que los estudiantes que utilizan el método AICLE mejoran su capacidad de escucha, la fluidez pero no la correc- 
ción. En esta conclusión coinciden otros estudios (este es el caso de Patsy y Lightbrown, en Vallbona, 2008).

\section{Metodología}

Para llevar a cabo la investigación, el equipo planteó una triangulación metodológica que se basa en la interrelación entre técnicas cualitativas (entrevistas semi-estructuradas) y cuantitativas (encuestas). La intersección entre ambas técnicas nos permite unir la rigurosidad de las cifras con la interpretación que posibilitan las respuestas complejas de las entrevistas semiestructuradas o en profundidad (De Miguel, 2005).

En primer lugar, se diseñaron dos encuestas para los estudiantes de segundo ciclo de la FEC: una para los que han cursado asignaturas AICLE y otra para los que no. Las encuestas se pasaron a estos estudiantes usando un muestreo aleatorio simple en ambos colectivos. La estructura de ambas encuestas era doble, con preguntas cerradas a responder según el grado de acuerdo (escala numérica, 0 a 4) o, también, preguntas de respuesta abierta.

Los contenidos de las encuestas se centraron en los siguientes aspectos: nivel de inglés, opinión sobre el AICLE, mejoras a implementar en las asignaturas AICLE y reticencias de los estudiantes.

En segundo lugar, las cifras se cruzaron con las valoraciones cualitativas de los profesores que han impartido clases AICLE durante el curso 2011-2012. El objetivo de estas entrevistas semiestructuradas era conseguir una valoración crítica del método AICLE por parte de los docentes, tanto en relación con la docencia dentro del grupo clase como en relación a la formación recibida por parte de la UVic antes de impartir este tipo de asignaturas ${ }^{2}$. Los profesores participantes en este estudio se escogieron usando una muestra tipológica o estratégica, con participación de profesores nativos (2) y no nativos (2), y con experiencia o no en las clases AICLE (2 y 2)

\begin{tabular}{|l|l|c|c|}
\hline Referencia & Asignatura AICLE & Primer año & Nativo \\
\hline Profesor 1 & Social Journalism & Sí & No \\
\hline Profesor 2 & Courrent Affairs Workshop & Sí & No \\
\hline Profesor 3 & Anglosaxon Journalism & No & Sí \\
\hline Profesor 4 & Formadora AILCE & No & Sí \\
\hline
\end{tabular}

Perfil de las personas entrevistadas en el trabajo de campo. Elaboración propia

Las entrevistas semiestructuradas con los docentes AICLE nos sirven para extraer datos cuantificables y comparables a partir "de una lista de ítems o temas que se quieren cubrir, pero sin tener un cuestionario formalizado que guie el orden o el contenido de las preguntas" (De Miguel, 2005. Pág. 253). La lista de bloques temáticos se estructuró siguiendo un orden lógico de contenidos, desde los antecedentes hasta el fu-

${ }^{2}$ Los profesores que impartieron asignaturas AICLE a partir del curso 2011-2012 recibieron un curso de formación de diez horas por parte del CIFE, el centro de formación del profesorado de la UVic. Este mismo organismo también validó los programas docentes de cada asignatura y, sobre todo, su metodología de impartición. 
turo, pasando por el panorama actual del AICLE en la UVic. Los bloques son: historia del AICLE en la UVic; preparación de las asignaturas AICLE; percepción sobre el funcionamiento de las asignaturas AICLE; relación profesor-alumno en las clases AICLE; propuestas de mejora en el funcionamiento AICLE.

Las transcripciones de las entrevistas fueron volcadas en una matriz de Excel, dónde fueron codificadas con el objetivo de poder gestionar mejor el contendio del trabajo de campo.

\section{Desarrollo. Los estudios de Ciencias de la Comunicación de la UVic y la apli- cación del AICLE}

En el segundo ciclo de los estudios de comunicación de la FEC, las únicas asignaturas en inglés son las vinculadas al intinerario de optativas internacional Business and Communication (60 ECTS) y, también, las tres que se ofertan concretamente en los estudios del grado en Periodismo (Current Affairs Workshop, Anglosaxon Journalism y Social Journalism). Aunque la oferta es muy nueva, y algunas asignaturas del grado en Periodismo "han de impartirse en catalán o español porque su contenido forma parte de la continuidad lógica de los estudios" (Profesor 2), también llega un momento

"en que los estudios de Periodismo han de iniciar un proceso de internacionalización y también han de tener una serie de asignaturas que se ofrezcan en inglés al margen de las de lengua" (Profesor 2)

De hecho, el fomento de la internacionalización a través de asignaturas AICLE podría dar un valor añadido a los estudios de la UVic, tal y como afirma el Profesor 3. Pero, impartir asignaturas AICLE implica no solo tener un buen nivel de inglés, sino dominar contenidos específicos de un grado. Y, en este aspecto, hay pocos profesores que puedan asumir el reto: en la FEC solo hay 6 profesores que imparten asignaturas AICLE.

En cuanto a los estudiantes de AICLE, la mayoría considera aceptable el nivel de inglés de los profesores. Ahora bien, 9 de 15 valoran en un 2 sobre 4 la oferta de asignaturas AICLE de la UVic. No obstante, sí que reconocen haber aprendido más inglés en la Universidad que en el bachillerato (10 de 15) y que las clases AICLE han contribuido a esta mejora del nivel de su inglés, sobre todo en lo referente a la expresión oral. Justamente, las clases AICLE focalizan la atención en la mejora de la expresión oral:

"Con el AICLE los estudiantes han de ser capaces de tener una conversación en inglés

de forma fluida, hacer preguntas o, por ejemplo, seguir una rueda de prensa en inglés" (Profesor 3)

Si bien los argumentos anteriores justifican la importancia que los profesores dan a las clases AICLE, los motivos por los cuales los estudiantes se matriculan en las asignaturas son diferentes: la mayoría cursan estas asignaturas "por obligación", es decir, por necesidad de complementar un determinado itinerario de optatividad. Ahora bien, la mitad de ellos indica que el hecho que sean asignaturas en inglés no les supuso un inconveniente a la hora de escogerlas. En relación a los que no han cursado asignaturas AICLE, resalta el hecho que 25 de ellos han indicado que no sabían qué era el Aprendizaje Integrado de Contenidos y Lenguas Extranjeras (AICLE). 


\subsection{Beneficios del AICLE}

Después del trabajo de campo, podemos afirmar que tanto para los docentes como para los estudiantes, la formación a través de asignaturas AICLE tiene más beneficios que inconvenientes.

No obstante, los profesores comentan que no puede confundirse una asignatura AICLE con una de lengua inglesa: en las asignaturas AICLE lo importante es el contenido, mientras que el aprendizaje del inglés se convierte en un valor añadido (sobre todo en lo referente a vocabulario específico y expresión oral). El Profesor 2 afirma, en este sentido:

"Creo que en Periodismo es muy importante que los estudiantes tengan habilidad para hacer preguntas en inglés; es decir, que no tengan miedo a trabajar en esta lengua. Por lo tanto, en Periodismo hemos intentado que todas las clases AICLE tengan un fuerte componente de formación de la expresión oral" (Profesor 2)

A parte de la mejora de la expresión oral, los profesores aseguran que la presencia de estudiantes internacionales en las clases enriquece los cursos: ya que hace que los autóctonos tengan que hacer más esfuerzo para expresarse correctamente.

Según el Profesor 2, hace falta remarcar que los estudiantes que entiendan el contenido de la asignatura aprobarán, más allá de su nivel de inglés. De hecho, según el Profesor 4, en una clase de contenidos en inglés los estudiantes tienen tendencia a prestar más atención para no perder el hilo argumental del contenido. Según este mismo profesor, que también es formador de AICLE, los estudiantes que antes de cursar asignaturas AICLE tienen un nivel B1, al final de sus estudios acaban con un nivel equivalente a un B2.

\subsection{Inconvenientes del AICLE}

La implementación del AICLE también recibe críticas. Los docentes consideran que su esfuerzo no es suficientemente reconocido por parte de la Univesidad-solo tienen un complemento salarial único, en función de los créditos impartidos en inglés. Esto se convierte en un problema importante, ya que la preparación de una asignatura AICLE requiere más tiempo: según ellos, casi el doble que el tiempo destinado a una asignatura impartida en catalán o español. El profesor 4 lo resume así:

"Estos profesores han hecho 10 horas de curso de formación sin cobrar nada, voluntariamente. El esfuerzo es mucho mayor y no tienen ninguna ventaja extra importante" (Profesor 4)

La falta de reconocimiento implica, según el Profesor 2, que "los profesores no quieren hacer estas asignaturas y cuesta encontrar gente que las quiera impartir".

Además, si bien el profesorado valora positivamente la convivencia en una misma clase de estudiantes nacionales e internacionales, reconocen que a veces es difícil lidiar con la coexistencia de perfiles tan diferentes de alumnos. De hecho, según las encuestas realizadas, tanto los estudiantes de asignaturas AICLE como los que no las han elegido, ven en la presencia de estudiantes del programa internacional un handicap que limita su participación. 
Otro inconveniente es cómo abordar la corrección del nivel de inglés que tienen los estudiantes. Si bien todos los profesores apuntan que lo oral es prioritario, existen más dudas sobre la manera de enfocar la corrección gramatical ya que no todos los profesores son especialistas en lengua ni tienen formación filológica. Según el profesor 2, de formación periodista,

"nuestra función tampoco es corregir la gramática. A lo mejor, el léxico es función nuestra, ya que el AICLE implica ofrecer al estudiante listas de vocabulario específico. Pero, en ningún caso el AICLE permite centrarse en la gramática porque tampoco eres profesor de lengua. Yo hago correcciones genéricas en lo relativo a la expresión escrita, pero sí que presto mucha más atención a la manera como presentan un programa de radio o televisión" (Profesor 2).

Otro inconveniente destacable es la falta de información. De las entrevistas y las encuestas se desprende que desde la FEC no se ha sabido "vender" del todo bien estas asignaturas a los estudiantes. Como se ha explicado anteriormente, la mayoría de estudiantes encuestados que no han cursado asignaturas AICLE afirma no saber de la existencia de éstas. No obstante, otra parte de estudiantes afirman no haber cursado estas asignaturas por miedo a no tener el nivel suficiente de inglés para aprobar.

\subsection{Soluciones y aspectos a mejorar}

En este punto se complementa lo dicho anteriormente. En primer lugar, la falta de reconocimiento -según los entrevistados- de la Universidad hacia los profesores AICLE requiere un análisis crítico del estado de la cuestión por parte de la dirección de la UVic: cuanto más reconocimiento, mejor se trabaja y más a gusto se preparan las clases. Igualmente, se ha de insistir en el control de la calidad de la docencia en las clases AICLE para mejorar los resultados obtenidos hasta el momento.

En segundo lugar, se ha visto que respecto la mezcla entre estudiantes internacionales y nacionales en los cursos no hay consenso entre alumnos y docentes. Partiendo de la base que la interacción es positiva, según los profesores, se requiere profundizar en las relaciones entre estudiantes como una de las bases del modelo AICLE. Uno de los argumentos que dan los estudiantes nacionales para poner en duda las virtudes de esta interacción entre estudiantes internacionales y nacionales es el miedo que tienen los españoles a "hacer el ridículo" y a no tener un nivel suficiente de inglés.

Finalmente, y al margen de todo esto, hace falta comentar la "aceptable" oferta de asignaturas AICLE que, según los profesores entrevistados, tiene la UVic en relación con otras universidades catalanas. No obstante, a ojos de los estudiantes, esta oferta aún tiene margen de mejora para poder englobar a muchas más áreas de especialización.

\section{Conclusiones}

Una vez analizados los resultados obtenidos la conclusión más evidente es que profesores y alumnos no coinciden en el grado de satisfacción sobre la oferta de asignaturas en inglés: mientras los docentes creen que es buena, los alumnos la consideran pobre. Las entrevistas con los docentes indican que aunque los planes de estudio han evolucionado en relación al AICLE, la oferta aún puede ser insuficiente para reforzar 
la internacionalización de la Universidad dentro del Espacio Europeo de Educación Superior (EEES).

En segundo lugar, destacar que algunos estudiantes han considerado que el nivel de aprendizaje en las asignaturas AICLE es menor que en las impartidas en catalán o español. Esto se debe al hecho que en una misma aula conviven estudiantes con diversos niveles de inglés, desde aquellos que vienen de escuelas donde la lengua extranjera era el francés hasta los estudiantes internacionales de países anglosajones. En estas circunstancias, el profesor ha de adaptar los contenidos a cada estudiante e impide un avance más rápido del programa académico. No obstante, la presencia de estudiantes ERASMUS en clase puede ser un beneficio. La interrelación entre estudiantes internacionales y nacionales permite a los segundos mejorar su nivel de inglés a través de los encuentros formales (en clase) o informales (a la hora de realizar trabajo en grupo). Mientras los profesores valoran muy positivamente la interrelación entre ambos colectivos, no pasa lo mismo con algunos estudiantes, que reconocen tener cierta pereza a la hora de relacionarse con los compañeros internacionales.

Finalmente, donde parece haber acuerdo entre docentes y estudiantes es en que las clases AICLE permiten una mejora de la fluidez del inglés oral. Durante los años que los estudiantes nacionales cursan estudios preuniversitarios la mayoría de asignaturas de inglés se focalizan en la gramática y el vocabulario, pero es difícil que trabajen correctamente las competencias vinculadas a la expresión oral. En las asignaturas AICLE, y tal y como metodológicamente se plantean en la UVic, la mejora de la expresión oral es el resultado de aprendizaje principal.

\section{Referencias bibliográficas}

ÁLVAREZ JIMENEZ, Mercedes et al. (2009): Materiales didácticos de apoyo para secciones bilingües de enseñanza secundaria. Oviedo, Consejería de Educación y Ciencia.

COYLE, Do (2005): "Developing CLIL: Towards a Theory of Practice", en APAC Monograph, $\mathrm{n}^{\circ}$ 6, Barcelona. Associació de Professors i Professore d'Anglès de Catalunya, pp. 5-29.

COYLE, Do; HOOD, Philip y MARSH, David (2010): CLIL: content and language integrated learning. New York, Cambridge University Press.

EF EPI (2009): EF EPI. Índice de nivel de inglés. Barcelona, EF Education First.

EURYDICE (2006): Key data on teaching languages at school in Europe. Bruxelles, Eurydice.

FLORIT y BALLESTER, Carme (2011): Caracterització de les pràctiques docents en AICLE: Una recerca en acció per a un ensenyament-aprenentatge integrat de continguts i llengua estrangera més efectiu. Barcelona, Pràctica Docent Efectiva AICLE.

MARSH, David; MARSLAND, Bruce; MALJERS, Anne (1998): Future Scenarios in Content and Language Integrated Learning. Jyväskylä, Continuing Education Centre University of Jyväskylä. 
MARSH, David y LANGÉ, Gisella (1999): Implementing Content and Language Integrated Learning. Jyväskylä, Continuing Education Centre University of Jyväskylä.

MIGUEL, Roberto de (2005): "La entrevista en profundidad a los emisores y receptores de los medios", en BERGANZA y SAN ROMAN (coord.): Investigar en comunicación. Madrid, McGraw Hill, pp. 251-263.

NAVÉS y NOGUÉS, Teresa (2007): Does Content and Language Integrated Learinig and Teaching have a future in our schools? Barcelona, Universitat de Barcelona.

VALLBONA, Anna (2008): Implementing CLIL in the Primary Classroom in Catalonia. MA Disertation. Barcelona, Bellaterra, Universitat Autònoma de Barcelona.

\section{Xavier GINESTA PORTET}

Universitat de Vic

Departamento de Comunicación

Doctor en Comunicación y Periodismo por la Universidad Autónoma de Barcelona. Profesor y Coordinador de la titulación de Periodismo y profesor xavier.ginesta@uvic.cat

\section{Gerard COLL-PLANAS}

Universitat de Vic

Doctor en Sociología por la Universidad Autónoma de Barcelona. Profesor del Departamento de Lenguas, Ciencias Sociales y Jurídicas

gerard.coll@uvic.cat

\section{Jordi DE SAN EUGENIO VELA}

Universitat de Vic

Doctor en Comunicación Social por la Universitat Pompeu Fabra. Director y profesor del Departamento de Comunicación

jordi.saneugenio@uvic.cat 